\section{Fatores associados à prática de atividade física global e de lazer em idosos: Inquérito de Saúde no Estado de São Paulo (ISA-SP), Brasil}

\author{
Factors associated with global and leisure-time \\ physical activity in the elderly: a health survey in \\ São Paulo (ISA-SP), Brazil
}

Maria Paula do Amaral Zaitune 1 Marilisa Berti de Azevedo Barros 1 Chester Luiz Galvão César 2 Luana Carandina 3 Moisés Goldbaum 4 Maria Cecilia Goi Porto Alves 5

\footnotetext{
1 Faculdade de Ciências Médicas, Universidade Estadual de Campinas, Campinas, Brasil.

${ }^{2}$ Faculdade de Saúde Pública, Universidade de São Paulo, São Paulo, Brasil. 3 Faculdade de Medicina de Botucatu, Universidade Estadual Paulista Júlio de Mesquita Filho, Botucatu, Brasil.

4 Faculdade de Medicina, Universidade de São Paulo, São Paulo, Brasil.

5 Secretaria de Estado da Saúde de São Paulo, São Paulo, Brasil.

Correspondência M. P. A. Zaitune Departamento de Medicina Preventiva e Social, Faculdade de Ciências Médicas, Universidade Estadual de Campinas. C. P. 6111, Campinas, $S P$ 13083-970, Brasil.

mpaula@fcm.unicamp.br
}

\begin{abstract}
The purpose of this study was to measure the prevalence of global and leisure-time physical activity and associated factors in the elderly. This was a population-based cross-sectional study covering a multiple-stage sample of 1,950 subjects 60 years or older living in areas of São Paulo State, Brazil. Prevalence of global physical activity (assessed through the short version of the International Physical Activity Questionnaire - IPAQ) was $73.9 \%$, and prevalence of leisure-time physical activity was $28.4 \%$. The results highlight the differences between factors associated with global and leisure-time physical activities. The social groups most prone to overall sedentary lifestyle and especially to lack of leisure-time physical activity should be the main targets of health policies aimed at promoting healthier lifestyles.
\end{abstract}

Motor Activity; Leisure Activities; Health of the Elderly

\section{Introdução}

O processo de envelhecimento é acompanhado por progressivas e importantes alterações fisiológicas em órgãos e sistemas como a diminuição e alteração da composição muscular, da massa óssea e da capacidade cardiorrespiratória que podem comprometer o desempenho de tarefas diversas, inclusive as mais simples do dia-a-dia 1. Embora seja apontado que doenças e limitações não são resultados inevitáveis do envelhecimento 2 , é amplamente evidenciado que a prevalência de múltiplas condições crônicas é mais elevada entre os idosos, existindo neste segmento etário grande heterogeneidade entre os indivíduos no que diz respeito às condições de saúde, à funcionalidade e às necessidades de cuidado ${ }^{3}$.

Recentemente, o Ministério da Saúde estabeleceu a Política Nacional de Atenção à Saúde da Pessoa Idosa, que propõe ações que favorecem modos de vida mais saudáveis no decorrer da vida 2. Uma das abordagens sugeridas para alcançar o envelhecimento saudável e ativo, e que assume um papel fundamental na promoção da saúde e nas diferentes estratégias de prevenção de doenças, é a prática regular de atividade física 4,5 .

Nas últimas décadas, a atividade física tem sido crescentemente promovida pelos reconhecidos benefícios físicos, psicológicos e sociais advindos da sua prática 4,6. Especialmente entre os idosos, favorece a manutenção da independên- 
cia, da saúde e da qualidade de vida e pode reduzir o uso de serviços de saúde e de medicamentos, o risco de desenvolver doenças ou agravos crônicos, de morte e de institucionalização 7,8,9.

Geralmente, os benefícios biológicos decorrentes da prática de atividade física apresentam um efeito dose-resposta, ou seja, a sobrecarga física imposta ao organismo provoca adaptações de órgãos e sistemas de modos distintos dependendo da intensidade, freqüência e duração do estímulo. Alguns benefícios podem ser alcançados mesmo quando há apenas um pequeno incremento na atividade em indivíduos sedentários 10.

A atividade física, caracterizada por qualquer movimento do corpo em decorrência da contração dos músculos esqueléticos, pode ser realizada em diferentes contextos: no trabalho, nos deslocamentos a pé ou de bicicleta, nas atividades domésticas e no lazer. A atividade física de lazer é, portanto, um componente da atividade física global e refere-se à atividade física praticada durante o tempo livre, ou seja, no tempo não empenhado em obrigações de trabalho e do lar e que se pode dispor livremente para a prática destas atividades que incluem exercícios e esportes, entre outras 11 .

Estudos têm verificado algumas dificuldades que permeiam o engajamento do idoso em atividade física, como: medo de queda ou lesão, medo de ser vítima de violência ao exercitar-se ao ar livre, sensação de cansaço, morbidades, limitação física, dor, falta de companhia ou de tempo para exercitar-se 12,13.

O conhecimento da prevalência de indivíduos fisicamente ativos e, sobretudo, daqueles com menor participação em atividade física de lazer norteia as propostas de incentivo à prática regular destas atividades, no sentido de atingir os segmentos mais sedentários e na expectativa de gerar melhor qualidade de saúde e de vida, e economia nos gastos com tratamentos médicos.

Poucos estudos avaliaram a prevalência de atividades físicas especificamente em idosos no Brasil 14,15,16. O presente trabalho objetiva analisar a prevalência da prática de atividade física global e de lazer em idosos e seus fatores associados, em áreas do Estado de São Paulo, buscando obter conhecimentos que possam contribuir para a formulação e avaliação das políticas públicas relativas à promoção do envelhecimento ativo.

\section{Métodos}

\section{População de estudo}

Trata-se de um estudo transversal, de base populacional, que incluiu indivíduos de 60 anos e mais, não institucionalizados, residentes em quatro áreas do Estado de São Paulo: (1) região sudoeste da Grande São Paulo, constituída pelos municípios de Taboão da Serra, Itapecerica da Serra e Embu; (2) Distrito do Butantã, no Município de São Paulo; (3) Município de Campinas e; (4) Município de Botucatu. Esta pesquisa é parte de um estudo multicêntrico - Inquérito de Saúde no Estado de São Paulo (ISA-SP), realizado em 2001-2002 17.

Para este inquérito foi estimado um tamanho mínimo de amostra de 196 pessoas para cada domínio de idade e sexo, de cada uma das quatro áreas estudadas. Para o cálculo do tamanho da amostra decidiu-se por utilizar uma estimativa mais conservadora, que define a variabilidade máxima dos dados, ou seja, uma prevalência de $50 \%$, a ser estimada com erro máximo de 0,07 , nível de $95 \%$ de confiança e efeito de delineamento de dois. Considerando uma possível perda de $20 \%$ foram selecionados 250 indivíduos em cada domínio. Os domínios amostrais foram: menor de 1 ano, 2-11 anos, 12-19 anos masculino, 12-19 anos feminino, 20-59 anos masculino, 20-59 anos feminino, 60 anos e mais masculino e 60 anos e mais feminino. Para o presente estudo foram incluídos apenas os domínios de 60 anos e mais, de ambos os sexos.

A amostragem foi probabilística, estratificada, por conglomerados e em dois estágios. Com base nos dados do Instituto Brasileiro de Geografia e Estatística (IBGE), os setores censitários foram classificados e agrupados em três estratos segundo o porcentual de chefes de família com nível universitário: menos de 5\%, de 5-25\% e mais de $25 \%$. De cada estrato, foram sorteados dez setores censitários, e após arrolamento para atualização de mapas e contagem das residências, sorteou-se domicílios e em cada um foram entrevistados indivíduos segundo os domínios definidos. Detalhes do procedimento amostral encontram-se em outra publicação 18 .

As informações foram obtidas por meio de questionário aplicado por entrevistadores treinados, diretamente do indivíduo selecionado. $\mathrm{O}$ questionário foi composto principalmente por questões fechadas, organizadas em blocos temáticos: características socioeconômicas, da família e do domicílio, estilo de vida, percepção subjetiva e qualidade de saúde, referência de morbidade nos 15 dias que antecederam a entrevista, check list de doenças crônicas, acidentes e violências, 
deficiência física, hospitalização, uso de outros serviços de saúde, consumo de medicamentos e gastos com saúde.

As variáveis incluídas no presente estudo foram: prática de atividade física no contexto global; prática de atividade física no contexto de lazer; demográficas; socioeconômicas; fatores comportamentais de risco à saúde e morbidades.

\section{Prática de atividade física no contexto global}

O nível de atividade física foi avaliado pelo Questionário Internacional de Atividade Física (QIAF), versão 8. Sob a chancela da Organização Mundial da Saúde (OMS), o QIAF foi proposto pelo Grupo Internacional para Consenso em Medidas da Atividade Física, constituído por 25 países, entre eles o Brasil, com o intuito de compor um instrumento único que pudesse estimar o nível de atividade física de populações de diferentes países. Existem duas versões do questionário, uma no formato longo e outra no curto 19,20. A versão simplificada do QIAF, usada neste estudo, é composta por sete questões e provê informações da freqüência e duração de caminhadas e de atividades de intensidades moderada e vigorosa realizadas em uma semana habitual, produzindo uma avaliação da atividade física global sem discriminá-la quanto ao contexto (trabalho, locomoção, doméstica e lazer) em que as atividades são realizadas 20 .

O instrumento permite classificar o indivíduo como:

a) Sedentário: se não realiza nenhum tipo de atividade física por pelo menos 10 minutos contínuos durante a semana;

b) Insuficientemente ativo: se realiza algum tipo de atividade física por pelo menos 10 minutos por semana, porém insuficiente para ser classificado como ativo;

c) Ativo: se realiza, em três ou mais dias da semana, atividade vigorosa de pelo menos 20 minutos por sessão ou realiza, em cinco ou mais dias da semana, atividade de intensidade moderada ou caminhada de pelo menos 30 minutos por sessão, ou realiza em cinco ou mais dias da semana qualquer combinação de atividades (caminhada + atividade moderada + atividade vigorosa), alcançando 150 minutos ou mais por semana;

d) Muito ativo: indivíduo que realiza, em cinco ou mais dias da semana, atividade vigorosa de pelo menos 30 minutos por sessão ou três ou mais dias por semana de atividade vigorosa de pelo menos 20 minutos por sessão, somada a cinco dias ou mais por semana de atividade moderada e/ou caminhada por pelo menos 30 minutos por sessão ${ }^{21}$.
De acordo com os propositores do instrumento, atividade leve significa a que exige pouco esforço físico e que praticamente não altera a respiração. A atividade moderada requer algum esforço físico e respiração um pouco mais intensa e, finalmente, a atividade vigorosa demanda um grande esforço físico e uma respiração muito mais intensa que a normal 21.

Para o presente estudo, considerou-se a análise por categorias, usando-se os agrupamentos: "ativos + muito ativos" e "sedentários + insuficientemente ativos".

\section{- Prática de atividade física no contexto de lazer}

A prática de atividade física no contexto de lazer foi definida como a prática regular de qualquer esporte ou exercício físico, pelo menos uma vez por semana. A pergunta feita ao entrevistado foi: "Pratica regularmente, pelo menos uma vez por semana, algum esporte ou exercício físico? Se sim, qual?". Essa questão permitia resposta de não praticar nenhum esporte ou mais de uma resposta quanto ao tipo de esporte ou exercício físico praticado.

\section{- Demográficas}

As variáveis demográficas foram: sexo, idade, cor/ raça, estado conjugal, naturalidade e religião.

\section{- Socioeconômicas}

As variáveis socioeconômicas foram: escolaridade, renda familiar mensal per capita (em salários mínimos) e atividade ocupacional.

\section{- Fatores comportamentais de risco à saúde}

As variáveis relacionadas aos fatores comportamentais de risco à saúde foram: freqüência semanal de ingestão de bebida alcoólica e tabagismo.

\section{- Morbidades}

As variáveis relacionadas às morbidades foram: número de doenças crônicas referidas; transtorno mental comum avaliado baseando-se no Self Reporting Questionnaire (SRQ-20), com ponto de corte $7 / 822 \mathrm{e}$; índice de massa corporal calculado com dados de peso e altura referidos (IMC = $\mathrm{kg} / \mathrm{m}^{2}$ ), sendo as categorias de IMC utilizadas as preconizadas para o idoso com os seguintes pontos de corte: baixo peso $\left(<22 \mathrm{~kg} / \mathrm{m}^{2}\right)$, eutrofia $\left(22 \leq \mathrm{IMC} \leq 27 \mathrm{~kg} / \mathrm{m}^{2}\right)$ e sobrepeso $\left(>27 \mathrm{~kg} / \mathrm{m}^{2}\right) 23$. 


\section{Análise dos dados}

Foram feitas estimativas de prevalências e testada a associação entre as diversas variáveis e a prática de atividade física global e de lazer usando-se o teste qui-quadrado $\left(\chi^{2}\right)$ com nível de significância de $5 \%$; foram calculadas as razões de prevalências brutas e ajustadas para idade e sexo e respectivos intervalos de $95 \%$ de confiança (IC95\%), usando-se regressão simples e múltipla de Poisson ${ }^{24}$. Apenas as variáveis que apresentaram o valor de $\mathrm{p}<0,20$ na análise bivariada foram incluídas no modelo de regressão múltipla, sendo mantidas no modelo aquelas que persistiram associadas com a variável dependente com valor de $\mathrm{p}<0,05$. Todas as análises foram feitas com o programa Stata 8.0 (Stata Corp., College Station, Estados Unidos), utilizando os comandos svy que permitem a incorporação dos fatores de ponderação da amostra, considerando além dos pesos também os estratos e os conglomerados. Os pesos finais incorporaram ainda ajustes de pós-estratificação que consideraram as informações do Censo Demográfico de 2000 (IBGE. http://www. ibge.gov.br) sobre estrutura da população por idade e sexo.

O projeto foi aprovado pelo Comitê de Ética em pesquisa da Faculdade de Ciências Médicas da Universidade Estadual de Campinas sob o parecer $n^{\circ} .369 / 2000$.

\section{Resultados}

Entre os idosos identificados nos domicílios sorteados, ocorreram $9,4 \%$ de perdas, sendo $9,1 \%$ de recusas e $0,3 \%$ por não obtenção da entrevista após mais de três tentativas. No total, foram entrevistados 1.950 indivíduos com idade igual ou superior a 60 anos, dos quais 1.026 eram do sexo feminino. A média de idade foi de 69,7 anos (IC95\%: 68,9-70,4).

A prevalência de ativos e muito ativos avaliados pelo QIAF foi de 73,9\% (IC95\%: 70,8-76,7\%), sendo maior entre as mulheres $(78,5 \%)$ do que nos homens $(67,7 \%)$ (Tabela 1$)$. Para a atividade física no lazer, encontrou-se baixa prevalência para ambos os sexos (28,4\%) (IC95\%: 24,8-32,3), porém, maior em homens $(32,3 \%)$ do que nas mulheres $(25,7 \%)$.

Em relação às variáveis sociodemográficas e econômicas (Tabelas 1 e 2) observou-se, após ajuste por idade e sexo, significativa maior prevalência de atividade física no contexto geral nos idosos: mais jovens (60-79 anos), em atividade ocupacional e, no limiar de significância estatística, para idosos com 4-7 anos de estudo e naturais de outros municípios e estados. Em relação à prática de atividade física de lazer, a prática mostrou-se mais freqüente nos indivíduos: brancos, não-evangélicos (católicos, outras religiões e sem religião), naturais de municípios do Estado de São Paulo, dos estratos de maior escolaridade (4 anos ou mais) e das categorias de melhor renda familiar (maior que 2,5 salários mínimos). A associação com estado conjugal, observada na razão de prevalência bruta, não persistiu após ajuste por idade e sexo.

Ao analisar a prevalência de ativos e muito ativos pelo QIAF segundo comportamentos relacionados à saúde e morbidades (Tabela 3), verificou-se que apresentam menor prevalência após ajuste por idade e sexo: idosos com quatro ou mais doenças crônicas, com transtorno mental comum e aqueles que não ingerem bebida alcoólica. A prevalência de ativos no lazer mostrouse significativamente mais elevada entre: idosos com sobrepeso, não-fumantes, que consomem bebidas alcoólicas com freqüência de 1-4 vezes por semana, com menos de quatro doenças crônicas e sem transtorno mental comum.

Na análise múltipla, usando-se a regressão múltipla de Poisson (Tabela 4), a atividade física global foi mais prevalente nas categorias: sexo feminino, faixa etária de 60-79 anos, naturalidade de outros municípios de São Paulo ou outros estados, menos de oito anos de escolaridade, ter atividade ocupacional, consumo de álcool 1-3 vezes por semana e ausência de transtorno mental comum. Para a atividade física no lazer, apresentaram significativa maior prevalência os indivíduos: com oito anos ou mais de escolaridade, renda de 2,5-4 salários mínimos, que não fumavam ou eram ex-fumantes, que consumiam bebida alcoólica de 1-3 vezes por semana e que não apresentavam transtorno mental comum.

\section{Discussão}

Este estudo traz informações sobre a prevalência de atividade física global e de lazer de idosos, e dos fatores socioeconômicos, demográficos e de saúde associados a esta prática. Os principais resultados obtidos foram: maior prevalência de ativos globalmente entre os idosos do sexo feminino, com idades de 60-79 anos, naturais de outros municípios de São Paulo ou outros estados, com menos de oito anos de escolaridade, com atividade ocupacional, que consumiam bebida alcoólica 1-3 vezes por semana e sem transtorno mental comum. Maior prevalência de idosos ativos no lazer foi encontrada nas categorias de oito anos ou mais de escolaridade, renda de 2,5-4 salários mínimos, que não fumavam ou eram exfumantes, que consumiam bebida alcoólica de 
Tabela 1

Prevalência e razões de prevalência (RP) de ativos e muito ativos nas atividades globais e de ativos no lazer, segundo variáveis sociodemográficas em pessoas com 60 anos ou mais. Inquérito de Saúde no Estado de São Paulo (ISA-SP), 2001-2002.

\begin{tabular}{|c|c|c|c|c|}
\hline \multirow[t]{2}{*}{ Variáveis e categorias } & \multicolumn{4}{|c|}{ Ativos e muito ativos (QIAF) } \\
\hline & $n$ * & Prevalência [\% (IC95\%)] & RP bruta (IC95\%) & $\begin{array}{l}\text { RP ajustada por idade e } \\
\text { sexo (IC95\%) }\end{array}$ \\
\hline Sexo & $p=0,0001 * \star$ & & & \\
\hline Masculino & 924 & $67,7(63,5-71,6)$ & 1,00 & 1,00 \\
\hline Feminino & 1.026 & $78,5(74,5-82,0)$ & $1,06(1,03-1,10)$ & $1,07(1,04-1,10)$ \\
\hline Total & 1.950 & $73,9(70,8-76,7)$ & & \\
\hline Faixa etária (anos) & $p=0,0000 * \star$ & & & \\
\hline $60-69$ & 1.089 & $78,6(75,1-81,8)$ & 1,00 & 1,00 \\
\hline $70-79$ & 642 & $73,4(68,5-77,7)$ & $0,97(0,94-1,00)$ & $0,97(0,94-1,00)$ \\
\hline $80 \mathrm{e}+$ & 219 & $50,4(39,8-61,0)$ & $0,84(0,78-0,90)$ & $0,84(0,78-0,90)$ \\
\hline Cor/Raça & $\mathrm{p}=0,1131 \star \star \star$ & & & \\
\hline Branca & 1.503 & $72,6(69,1-75,9)$ & 1,00 & 1,00 \\
\hline Outras & 440 & $77,9(72,1-82,8)$ & $1,03(0,99-1,07)$ & $1,02(0,98-1,05)$ \\
\hline Situação conjugal & $\mathrm{p}=0,1641 \star *$ & & & \\
\hline Com cônjuge & 1.167 & $75,5(71,7-78,9)$ & 1,00 & 1,00 \\
\hline Sem cônjuge & 772 & $71,6(66,8-76,0)$ & $0,98(0,95-1,01)$ & $0,97(0,93-1,00)$ \\
\hline Religião & $p=0,9779 * \star$ & & & \\
\hline Católica & 1.422 & $74,0(70,4-77,3)$ & 1,00 & 1,00 \\
\hline Evangélica & 305 & $74,2(65,6-81,2)$ & $1,00(0,95-1,05)$ & $0,99(0,94-1,04)$ \\
\hline Outras/Sem religião & 201 & $72,9(62,2-81,6)$ & $0,99(0,94-1,05)$ & $0,99(0,94-1,05)$ \\
\hline Naturalidade & $p=0,0643 * \star$ & & & \\
\hline Próprio município & 369 & $68,0(60,8-74,4)$ & 1,00 & 1,00 \\
\hline Outros municípios de São Paulo & 791 & $74,2(69,9-78,2)$ & $1,04(1,00-1,07)$ & $1,03(1,00-1,07)$ \\
\hline Outros estados & 781 & $76,4(72,4-79,9)$ & $1,05(1,00-1,10)$ & $1,04(1,00-1,09)$ \\
\hline \multirow[t]{2}{*}{ Variáveis e categorias } & \multicolumn{4}{|c|}{ Ativos no lazer } \\
\hline & $n$ * & Prevalência [\% (IC95\%)] & RP bruta (IC95\%) & $\begin{array}{c}\text { RP ajustada por idade e } \\
\text { sexo (IC95\%) }\end{array}$ \\
\hline \multicolumn{5}{|l|}{ Sexo } \\
\hline Masculino & 924 & $32,3(28,2-36,8)$ & 1,00 & 1,00 \\
\hline Feminino & 1.026 & $25,7(21,0-30,5)$ & $0,80(0,64-0,99)$ & $0,80(0,64-1,00)$ \\
\hline Total & 1.950 & $28,4(24,8-32,3)$ & & \\
\hline Faixa etária (anos) & $p=0,0930 * \star$ & & & \\
\hline $60-69$ & 1.088 & $29,0(24,7-33,7)$ & 1,00 & 1,00 \\
\hline $70-79$ & 642 & $30,2(25,8-35,0)$ & $1,03(0,86-1,22)$ & $1,05(0,88-1,25)$ \\
\hline $80 \mathrm{e}+$ & 220 & $19,7(12,5-29,8)$ & $0,68(0,44-1,04)$ & $0,69(0,45-1,06)$ \\
\hline Cor/Raça & $p=0,0009 * *$ & & & \\
\hline Branca & 1.505 & $31,1(27,3-35,1)$ & 1,00 & 1,00 \\
\hline Outras & 438 & $18,4(13,3-24,8)$ & $0,59(0,42-0,81)$ & $0,57(0,41-0,79)$ \\
\hline Situação conjugal & $\mathrm{p}=0,0303 * *$ & & & \\
\hline Com cônjuge & 1.167 & $30,8(27,0-34,7)$ & 1,00 & 1,00 \\
\hline Sem cônjuge & 772 & $25,1(20,5-30,4)$ & $0,81(0,67-0,98)$ & $0,90(0,72-1,11)$ \\
\hline Religião & $p=0,0008 * \star$ & & & \\
\hline Católica & 1.422 & $29,7(26,3-33,4)$ & 1,00 & 1,00 \\
\hline Evangélica & 304 & $18,1(12,8-25,1)$ & $0,61(0,45-0,83)$ & $0,61(0,45-0,83)$ \\
\hline Outras/Sem religião & 202 & $34,2(26,2-43,1)$ & $1,15(0,90-1,46)$ & $1,13(0,88-1,44)$ \\
\hline Naturalidade & $\mathrm{p}=0,0324 * *$ & & & \\
\hline Próprio município & 369 & $33,0(26,9-39,7)$ & 1,00 & 1,00 \\
\hline Outros municípios de São Paulo & 790 & $31,2(25,8-37,2)$ & $0,95(0,71-1,26)$ & $0,95(0,72-, 25)$ \\
\hline Outros estados & 782 & $23,6(19,0-28,9)$ & $0,72(0,55-0,93)$ & $0,70(0,54-0,91)$ \\
\hline
\end{tabular}

IC95\%: intervalo de 95\% de confiança; QIAF: Questionário Internacional de Atividade Física.

* Número de indivíduos na amostra não ponderada;

** Valor de $p$ do teste $\chi^{2}$. 
Tabela 2

Prevalência e razões de prevalência (RP) de ativos e muito ativos nas atividades globais e de ativos no lazer, segundo variáveis socioeconômicas em pessoas com 60 anos ou mais. Inquérito de Saúde no Estado de São Paulo (ISA-SP), 2001-2002.

\begin{tabular}{|c|c|c|c|c|}
\hline \multirow[t]{2}{*}{ Variáveis e categorias } & \multicolumn{4}{|c|}{ Ativos e muito ativos (QIAF) } \\
\hline & $n$ * & Prevalência [\% (IC95\%)] & RP bruta (IC95\%) & $\begin{array}{l}\text { RP ajustada por idade e } \\
\text { sexo (IC95\%) }\end{array}$ \\
\hline Escolaridade do indivíduo (anos) & $p=0,0027 \star \star$ & & & \\
\hline $0-3$ & 841 & $74,1(70,1-77,8)$ & 1,00 & 1,00 \\
\hline $4-7$ & 650 & $79,7(74,5-84,1)$ & $1,03(1,00-1,07)$ & $1,03(1,00-1,06)$ \\
\hline $8-11$ & 313 & $68,7(62,2-74,6)$ & $0,97(0,93-1,01)$ & $0,97(0,93-1,01)$ \\
\hline 12 ou + & 138 & $62,1(49,8-73,0)$ & $0,93(0,86-1,00)$ & $0,93(0,86-1,00)$ \\
\hline Renda per capita (salários mínimos) & $p=0,1468 * \star$ & & & \\
\hline$\leq 1$ & 565 & $74,2(69,4-78,5)$ & 1,00 & 1,00 \\
\hline$>1-2,5$ & 619 & $76,3(71,3-80,7)$ & $1,01(0,97-1,05)$ & $1,01(0,97-1,05)$ \\
\hline$>2,5-4$ & 304 & $77,2(69,8-83,3)$ & $1,02(0,98-1,06)$ & $1,02(0,98-1,06)$ \\
\hline$>4$ & 462 & $68,0(60,1-75,1)$ & $0,96(0,91-1,02)$ & $0,97(0,92-1,01)$ \\
\hline Em atividade ocupacional & $p=0,0058 * \star$ & & & \\
\hline Não & 1.449 & $71,8(68,1-75,2)$ & 1,00 & 1,00 \\
\hline Sim & 501 & $80,4(75,2-84,8)$ & $1,05(1,02-1,08)$ & $1,05(1,02-1,09)$ \\
\hline \multirow[t]{2}{*}{ Variáveis e categorias } & \multicolumn{4}{|c|}{ Ativos no lazer } \\
\hline & $n$ * & Prevalência [\% (IC95\%)] & RP bruta (IC95\%) & $\begin{array}{c}\text { RP ajustada por idade e } \\
\text { sexo (IC95\%) }\end{array}$ \\
\hline Escolaridade do indivíduo (anos) & $p=0,0000 * \star$ & & & \\
\hline $0-3$ & 840 & $19,9(15,9-24,6)$ & 1,00 & 1,00 \\
\hline $4-7$ & 650 & $27,9(22,5-34,0)$ & $1,39(1,04-1,85)$ & $1,38(1,04-1,83)$ \\
\hline $8-11$ & 314 & $42,3(34,1-51,0)$ & $2,17(1,60-2,96)$ & $2,14(1,57-2,91)$ \\
\hline $12 \mathrm{ou}+$ & 138 & $48,1(36,9-59,5)$ & $2,42(1,75-3,35)$ & $2,32(1,67-3,22)$ \\
\hline Renda per capita (salários mínimos) & $p=0,0000 * \star$ & & & \\
\hline$\leq 1$ & 566 & $19,0(15,2-23,7)$ & 1,00 & 1,00 \\
\hline$>1-2,5$ & 618 & $23,1(18,1-29,1)$ & $1,21(0,91-1,61)$ & $1,20(0,90-1,59)$ \\
\hline$>2,5-4$ & 303 & $38,5(31,2-46,3)$ & $2,06(1,50-2,82)$ & $2,09(1,53-2,86)$ \\
\hline$>4$ & 463 & $38,7(32,0-45,8)$ & $2,01(1,49-2,73)$ & $2,01(1,48-2,74)$ \\
\hline Em atividade ocupacional & $p=0,9770 * \star$ & & & \\
\hline Não & 1.451 & $28,4(24,2-32,9)$ & 1,00 & 1,00 \\
\hline Sim & 499 & $28,5(23,3-34,3)$ & $1,0(0,78-1,27)$ & $0,88(0,68-1,16)$ \\
\hline
\end{tabular}

IC95\%: intervalo de 95\% de confiança; QIAF: Questionário Internacional de Atividade Física.

* Número de indivíduos na amostra não ponderada;

** Valor de $p$ do teste $\chi^{2}$.

1-3 vezes por semana e que não apresentavam transtorno mental comum.

A baixa prevalência de ativos no lazer em idosos, observada em nosso estudo $(28,4 \%)$ foi um pouco superior à observada em pesquisas realizadas em São Paulo (21,3\%) 25, Florianópolis, Santa Catarina (25,7\%) 26 e Salvador, Bahia $(22,3 \%) 27$ para a mesma faixa de idade, e pouco inferior à média observada em 15 países europeus (33\%) ${ }^{28}$ para a população com 65 anos ou mais. No entanto, os resultados encontrados em nossa pesquisa foram bem distintos dos observa- dos no Inquérito de Fatores de Risco de Doenças Crônicas Não Transmissíveis por Entrevistas Telefônicas (VIGITEL) ${ }^{29}$, que encontrou prevalência de $12,7 \%$ em práticas de atividades físicas de lazer em idosos acima de 65 anos em capitais brasileiras. A possível explicação seriam as definições de atividade física de lazer que são distintas nas duas pesquisas. Pela definição de atividade física de lazer adotada neste estudo, indivíduos que praticam algum exercício físico ou esporte ao menos uma vez na semana não foram considerados sedentários, embora possam ser insufi- 
Tabela 3

Prevalência e razões de prevalência (RP) de ativos e muito ativos nas atividades globais e de ativos no lazer, segundo comportamentos de saúde e morbidades em pessoas com 60 anos ou mais. Inquérito de Saúde no Estado de São Paulo (ISA-SP), 2001-2002.

\begin{tabular}{|c|c|c|c|c|}
\hline \multirow[t]{2}{*}{ Variáveis e categorias } & \multicolumn{4}{|c|}{ Ativos e muito ativos (QIAF) } \\
\hline & $n$ * & Prevalência [\% (IC95\%)] & RP bruta (IC95\%) & $\begin{array}{l}\text { RP ajustada por idade } \\
\text { e sexo (IC95\%) }\end{array}$ \\
\hline Hábito de fumar & $p=0,0479 * *$ & & & \\
\hline Nunca fumou & 1.040 & $76,7(72,3-80,6)$ & 1,00 & 1,00 \\
\hline Fumante atual & 290 & $70,1(63,1-76,2)$ & $0,96(0,92-1,01)$ & $0,97(0,93-1,01)$ \\
\hline Ex-fumante & 618 & $70,3(65,5-74,7)$ & $0,96(0,93-1,00)$ & $0,99(0,95-1,02)$ \\
\hline $\begin{array}{l}\text { Freqüência de ingestão de bebida alcoólica } \\
\text { (vezes/semana) }\end{array}$ & $p=0,1264^{\star \star}$ & & & \\
\hline Não bebe & 1.214 & $71,5(67,3-75,3)$ & 1,00 & 1,00 \\
\hline Menos de 1 & 243 & $76,7(69,3-82,8)$ & $1,03(0,99-1,08)$ & $1,04(1,00-1,08)$ \\
\hline $1-3$ & 280 & $78,5(72,7-83,3)$ & $1,04(1,00-1,08)$ & $1,06(1,02-1,10)$ \\
\hline $4-7$ & 185 & $77,1(68,5-83,9)$ & $1,03(0,99-1,08)$ & $1,07(1,02-1,11)$ \\
\hline Índice de massa corporal & $p=0,6415^{\star \star}$ & & & \\
\hline Eutrofia $\left(22 \leq \mathrm{IMC} \leq 27 \mathrm{~kg} / \mathrm{m}^{2}\right)$ & 784 & $74,2(70,0-78,0)$ & 1,00 & 1,00 \\
\hline Baixo peso $\left(<22 \mathrm{~kg} / \mathrm{m}^{2}\right)$ & 294 & $77,7(69,9-84,0)$ & $1,02(0,97-1,07)$ & $1,03(0,98-1,07)$ \\
\hline Sobrepeso (> 27kg/m²) & 578 & $75,5(70,6-79,9)$ & $1,00(0,98-1,04)$ & $0,99(0,96-1,03)$ \\
\hline Número de doenças crônicas & $p=0,1292 * \star$ & & & \\
\hline $0-1$ & 757 & $76,2(71,3-80,5)$ & 1,00 & 1,00 \\
\hline $2-3$ & 696 & $74,0(69,6-77,9)$ & $0,99(0,95-1,02)$ & $0,97(0,94-1,01)$ \\
\hline 4 ou mais & 392 & $68,8(62,4-74,6)$ & $0,96(0,91-1,00)$ & $0,94(0,90-0,98)$ \\
\hline Transtorno mental comum & $p=0,0150 * \star$ & & & \\
\hline Ausente & 1.511 & $78,1(75,1-80,9)$ & 1,00 & 1,00 \\
\hline Presente & 380 & $67,4(57,6-76,0)$ & $0,94(0,89-0,99)$ & $0,93(0,88-0,98)$ \\
\hline \multirow[t]{2}{*}{ Variáveis e categorias } & \multicolumn{4}{|c|}{ Ativos no lazer } \\
\hline & $n$ * & Prevalência [\% (IC95\%)] & RP bruta (IC95\%) & $\begin{array}{l}\text { RP ajustada por idade } \\
\text { e sexo (IC95\%) }\end{array}$ \\
\hline Hábito de fumar & $p=0,0001 * \star$ & & & \\
\hline Nunca fumou & 1.039 & $29,7(25,3-34,6)$ & 1,00 & 1,00 \\
\hline Fumante atual & 290 & $13,8(9,6-19,3)$ & $0,46(0,31-0,68)$ & $0,41(0,27-0,60)$ \\
\hline Ex-fumante & 619 & $31,8(26,5-37,6)$ & $1,06(0,87-1,30)$ & $0,94(0,76-1,17)$ \\
\hline $\begin{array}{l}\text { Freqüência de ingestão de bebida alcoólica } \\
\text { (vezes/semana) }\end{array}$ & $p=0,0000 * *$ & & & \\
\hline Não bebe & 1.215 & $23,1(19,3-27,3)$ & 1,00 & 1,00 \\
\hline Menos de 1 & 243 & $32,7(25,9-40,3)$ & $1,47(1,13-1,92)$ & $1,44(1,11-1,88)$ \\
\hline $1-3$ & 279 & $44,7(37,1-52,6)$ & $1,95(1,56-2,43)$ & $1,85(1,46-2,34)$ \\
\hline $4-7$ & 185 & $29,0(22,4-36,8)$ & $1,26(0,95-1,68)$ & $1,17(0,88-1,56)$ \\
\hline Índice de massa corporal & $p=0,0010 * *$ & & & \\
\hline Eutrofia $\left(22 \leq \mathrm{IMC} \leq 27 \mathrm{~kg} / \mathrm{m}^{2}\right)$ & 784 & $35,4(29,9-41,5)$ & 1,00 & 1,00 \\
\hline Baixo peso $\left(<22 \mathrm{~kg} / \mathrm{m}^{2}\right)$ & 294 & $19,6(14,3-26,1)$ & $0,55(0,39-0,77)$ & $0,55(0,39-0,77)$ \\
\hline Sobrepeso (> 27kg/m²) & 577 & $30,9(26,1-36,1)$ & $0,87(0,70-1,08)$ & $0,87(0,71-1,07)$ \\
\hline Número de doenças crônicas & $p=0,0170 * \star$ & & & \\
\hline $0-1$ & 758 & $32,7(27,5-38,4)$ & 1,00 & 1,00 \\
\hline $2-3$ & 696 & $29,1(24,0-34,8)$ & $0,89(0,71-1,11)$ & $0,90(0,72-1,13)$ \\
\hline 4 ou mais & 392 & $20,8(15,6-27,3)$ & $0,66(0,48-0,91)$ & $0,69(0,50-0,94)$ \\
\hline Transtorno mental comum & $p=0,0000 * *$ & & & \\
\hline Ausente & 1.512 & $32,7(28,6-37,1)$ & 1,00 & 1,00 \\
\hline Presente & 379 & $15,8(11,7-21,1)$ & $0,48(0,35-0,65)$ & $0,50(0,37-0,67)$ \\
\hline
\end{tabular}

IC95\%: intervalo de 95\% de confiança; QIAF: Questionário Internacional de Atividade Física.

* Número de indivíduos na amostra não ponderada;

** Valor de $p$ do teste $\chi^{2}$ 
Modelo de regressão múltipla de Poisson: variáveis associadas à atividade física global e de lazer em idosos. Inquérito de Saúde no Estado de São Paulo (ISA-SP), 2001-2002.

\begin{tabular}{|c|c|c|}
\hline Variáveis e categorias & $\begin{array}{l}\text { Atividade física geral } \\
\text { RP (IC95\%) }\end{array}$ & $\begin{array}{c}\text { Atividade física de lazer } \\
\text { RP (IC95\%) }\end{array}$ \\
\hline \multicolumn{3}{|l|}{ Sexo } \\
\hline Masculino & 1,00 & \\
\hline Feminino & $1,09(1,06-1,13)$ & \\
\hline \multicolumn{3}{|l|}{ Faixa etária (anos) } \\
\hline $60-69$ & 1,00 & \\
\hline 70-79 & $0,98(0,95-1,00)$ & \\
\hline 80 e + & $0,89(0,84-0,95)$ & \\
\hline \multicolumn{3}{|l|}{ Naturalidade } \\
\hline Próprio município & 1,00 & \\
\hline Outros municípios de São Paulo & $1,04(1,01-1,07)$ & \\
\hline Municípios de outros estados & $1,04(1,00-1,09)$ & \\
\hline \multicolumn{3}{|l|}{ Escolaridade do indivíduo (anos) } \\
\hline $0-3$ & 1,00 & 1,00 \\
\hline $4-7$ & $1,03(1,00-1,06)$ & $1,21(0,94-1,56)$ \\
\hline $8-11$ & $0,96(0,92-0,99)$ & $1,57(1,15-2,15)$ \\
\hline $12 \mathrm{ou}+$ & $0,90(0,84-0,97)$ & $1,66(1,20-2,28)$ \\
\hline \multicolumn{3}{|l|}{ Renda per capita (em salários mínimos) } \\
\hline$\leq 1$ & & 1,00 \\
\hline$>1-2,5$ & & $1,04(0,79-1,35)$ \\
\hline$>2,5-4$ & & $1,51(1,15-1,99)$ \\
\hline$>4$ & & $1,33(0,96-1,83)$ \\
\hline \multicolumn{3}{|l|}{ Em atividade ocupacional } \\
\hline Não & 1,00 & \\
\hline Sim & $1,04(1,01-1,07)$ & \\
\hline \multicolumn{3}{|c|}{ Freqüência de ingestão de bebida alcoólica (vezes/semana) } \\
\hline Não bebe & 1,00 & 1,00 \\
\hline Menos de 1 & $1,03(0,99-1,06)$ & $1,27(0,97-1,65)$ \\
\hline $1-3$ & $1,06(1,02-1,10)$ & $1,50(1,15-1,97)$ \\
\hline $4-7$ & $1,05(0,99-1,10)$ & $1,14(0,88-1,47)$ \\
\hline \multicolumn{3}{|l|}{ Hábito de fumar } \\
\hline Não-fumante & & 1,00 \\
\hline Fumante & & $0,49(0,34-0,70)$ \\
\hline Ex-fumante & & $1,08(0,91-1,29)$ \\
\hline \multicolumn{3}{|l|}{ Transtorno mental comum } \\
\hline Ausente & 1,00 & 1,00 \\
\hline Presente & $0,93(0,88-0,98)$ & $0,59(0,43-0,81)$ \\
\hline
\end{tabular}

IC95\%: intervalo de 95\% de confiança; RP: razão de prevalência.

cientemente ativos, portanto, espera-se que os nossos achados de prevalência de ativos no lazer sejam superiores aos encontrados no VIGITEL, que considerou atividade física suficiente no lazer a prática de pelo menos 30 minutos diários de atividade física de intensidade leve ou moderada em cinco ou mais dias da semana, ou a prática de pelo menos 20 minutos diários de atividade física de intensidade vigorosa em três ou mais dias da semana.

A prevalência de ativos no contexto geral (73,9\%) mostrou-se superior à encontrada em estudo sobre atividade física e saúde mental realizada em idosos de Florianópolis (59,3\%), que usou a versão longa do QIAF 26 , e em pesquisa desenvolvida nas regiões Sul e Nordeste do Brasil 
(58\% em idosos de 65 anos e mais) 16 , que usou a versão curta do instrumento.

Vale lembrar que a atividade física de lazer é componente da global, o que justificaria a menor prevalência da primeira quando comparada à segunda. Apesar da atividade física de lazer ser uma das dimensões avaliadas pela atividade física global, elas usualmente apresentam diferenças nas associações com outras variáveis, pois a atividade física global sofre maior influência da atividade doméstica e da realizada no trabalho ${ }^{30}$.

Quando outros contextos (deslocamentos e atividades doméstica e ocupacional) são considerados, é comum observar maior prevalência de mulheres ativas quando comparadas aos homens ${ }^{30}$, assim como em nosso estudo $(78,5 \%$ das mulheres e $67,7 \%$ dos homens ativos globalmente). Por outro lado, quando considerado apenas o lazer, nossos resultados apontam que os homens são mais ativos do que as mulheres (32,3\% e $25,7 \%$, respectivamente), assim como verificado em outras pesquisas realizadas com adultos 16,31,32. Para explicar as diferenças nas práticas de atividade física segundo gênero, autores destacam as distintas atribuições nas responsabilidades familiares, bem como na função ocupacional desempenhada por homens e mulheres 33,34, além da existência de diferentes padrões sociais e culturais na adesão à prática de esporte 30,34,35

A diminuição dos níveis de atividade física no contexto geral e no lazer, na medida em que a idade aumenta, tem sido relatada em vários estudos $15,36,37,38$. O menor engajamento em atividades econômicas, como ocupações que requeiram alto dispêndio energético pela contração dos músculos esqueléticos e o trabalho realizado nas tarefas domésticas, poderiam explicar menor requisição do corpo neste segmento da população. Outros fatores decorrentes da idade também têm sido apontados como dificultadores para a prática de atividade física por idosos, sendo os principais: medo de machucar-se, sentir-se cansado ou muito velho, possuir doenças ou incapacidade física, não ter companhia ou não ter tempo para realizar exercícios físicos 12,13.

Sem atividade ocupacional poderia haver um acréscimo na prevalência de atividade no lazer dos idosos, mas não é o que comumente se observa 38 . Os benefícios biológicos, psicológicos e sociais advindos da prática de atividade física têm sido amplamente documentados inclusive para os idosos com mais idades, 6 e podem ser obtidos por meio das várias estratégias propostas para alcançar um envelhecimento com bemestar. É recomendado ainda que seja levado em consideração o prazer em realizar determinada atividade, além de observar outras características biopsicossociais no momento da prescrição ou escolha de exercícios, para maior adesão às práticas corporais por idosos 6 .

Assim como em nossa pesquisa, outros estudos verificaram que o nível socioeconômico está associado positivamente à atividade física de lazer 28,35,39 e inversamente à atividade física global 40. Provavelmente, aqueles com piores condições socioeconômicas deslocam-se mais a pé ou de bicicleta e/ou estão inseridos em atividades ocupacionais que requerem esforços de maior intensidade ${ }^{30}$, participam mais de atividades domésticas e têm menor oportunidade de participação em atividades físicas de lazer 32,37. Além disso, estudos mostram que idosos de diferentes níveis socioeconômicos percebem as barreiras para a prática de atividade física de maneiras distintas. Pessoas de segmentos de menor renda e escolaridade teriam menos acesso a equipamentos, a locais públicos adequados e seguros para a prática de exercício ou a ambientes e locais privados pagos e/ou de alto custo, ao conhecimento sobre atividade física e saúde, às orientações de profissionais de saúde e às condições de vida necessárias para incorporação de práticas consideradas saudáveis 13,27 .

Constatamos que idosos com baixo peso apresentaram prevalência de serem ativos no lazer inferior aos de peso normal e aos com sobrepeso, mas a significância estatística deixou de existir quando a razão de prevalências foi ajustada pelas demais variáveis do modelo de regressão múltipla. Análises com informação referida de peso e altura tendem a subestimar as prevalências de obesidade, embora pesquisas de validação apontem razoáveis valores de sensibilidade $\mathrm{e}$ especificidade 41 . Entretanto, as informações obtidas de pessoas idosas são menos válidas, pois é sugerido que os idosos confiram o peso e a altura com menor constância, levando ao desconhecimento dos verdadeiros valores destas medidas e, conseqüentemente, à pior estimativa do IMC para esta faixa etária em comparação aos mais jovens ${ }^{42}$. No presente estudo, utilizamos as categorias de IMC preconizadas para o idoso, pois os pontos de corte levam em consideração as mudanças na composição corporal (diminuição da água corporal e das massas óssea e muscular, e aumento e redistribuição da gordura corporal) decorrentes do envelhecimento 29.

Idosos naturais de outros estados mostraramse significativamente mais ativos globalmente e menos ativos no lazer mesmo após ajuste por idade e sexo, embora esta variável não tenha permanecido no modelo final. Em geral, pessoas que procedem de outros municípios ou estados têm em média menor nível socioeconômico e costumam e manter, por certo tempo, os hábitos e práticas culturais de seus locais de origem. 
Encontrou-se que os idosos fumantes apresentaram maior probabilidade de não estarem ativos no lazer, não havendo diferença na atividade física global segundo a condição tabágica. A maior razão de prevalência de atividade física de lazer verificada entre os ex-fumantes pode ser explicada pelo interesse ou mesmo a necessidade de aderir a práticas mais saudáveis por aqueles que deixaram de fumar. A associação entre inatividade e outros comportamentos não saudáveis (tabagismo, etilismo e dieta inadequada) é relatada na literatura e evidencia a tendência de coexistência destes comportamentos em subgrupos da população $43,44,45$, apontando para a necessidade de intervenções mais amplas dirigidas ao conjunto dos comportamentos e práticas relativas à saúde.

Observou-se associação positiva entre as práticas de atividade física global e de lazer e o consumo de bebida alcoólica em freqüência de 1-3 vezes por semana. Análise do Behavioral Risk Factor Surveillance System, com 264.684 adultos americanos, também evidenciou forte associação do consumo moderado de álcool com a prática de atividade física, mesmo depois de ajustada para idade, sexo, raça e escolaridade 46 . Outros estudos também apontam que o consumo moderado de bebida está associado à melhor percepção de saúde 47, à menor mortalidade e à menor incidência de algumas doenças crônicas 48 . O consumo moderado de álcool pode, entretanto, atuar como um marcador da boa condição física do idoso, indicando ausência de doença grave ou de doença que impeça o consumo de bebida alcoólica.

Outro fator que se mostrou associado, mas inversamente à atividade física de lazer e global, foi o transtorno mental comum. A literatura parece sustentar a contribuição significante que a atividade física e o exercício físico têm para o controle e para a diminuição do risco de depressão, de ansiedade e de outros transtornos de saúde mental 49,50, pois a atividade física atuaria nos mecanismos neuroendócrinos aumentando a secreção de endorfina e serotonina que poderiam resultar em melhora da ansiedade e do humor. Esse achado aponta a importância de avaliar e considerar a saúde mental do idoso para ajudá-lo no engajamento às práticas de atividade física, buscando superar os limites e a resistência que a depressão e a ansiedade poderiam provocar.

É importante lembrar que estudos transversais estão sujeitos ao viés de causalidade reversa, uma vez que exposição e os fatores são coletados em um mesmo momento, não permitindo identificar associações causais. Esse cuidado é fundamental ao considerarmos as associa- ções da atividade física com o consumo de álcool e com a presença de transtornos mentais comuns. Outra limitação deste estudo é o uso de informação referida da prática de atividade física por meio de questionário e não pelo uso de equipamentos de mensuração, o que confere maior margem de erro quando comparados a medidas diretas do nível de atividade física 51. Pesquisas apontam que a informação referida pode superestimar a prevalência de fisicamente ativos por ser um comportamento socialmente desejável 52,53. A vantagem da utilização de questionários auto-respondidos ou aplicados por entrevistador está no baixo custo e na rapidez para obtenção dos dados quando comparados a outros métodos 43,51,54. O QIAF foi submetido a estudos de validação em outros países e no Brasil 19. Os estudos de validação do QIAF para homens com 60 anos e mais 19 e para mulheres idosas 42 no Brasil foram feitos para a versão longa do instrumento, e ambos utilizaram como referência o pedômetro e o diário de atividade física. A reprodutibilidade mostrou correlação relativamente alta para ambos os sexos $(0,77$ e 0,95, para mulheres e homens idosos, respectivamente). Quanto aos indicadores de validade concorrente, observaram-se baixos índices de correlação entre as medidas obtidas usando-se o IPAQ e o pedômetro (mulheres com 60 anos e mais: índice de kappa $=0,27$; correlação de Spearman =0,27) (homens de 60 anos e mais: índice de kappa =0,19; correlação de Spearman =0,24) e IPAQ e o diário de atividade física (mulheres com 60 anos e mais: índice de kappa =0,37; correlação de Spearman =0,54) (homens de 60 anos e mais: índice de kappa =0,04 e correlação de Spearman $=0,38$ ) e, portanto, mostrando-se um instrumento com boa estabilidade de medidas e acurácia aceitável para uso em estudos epidemiológicos nas faixas etárias avaliadas. Ainda em relação ao instrumento, a versão curta do QIAF para a avaliação da atividade física global não permite identificar em que contexto específico as atividades foram realizadas, mas tem vantagens em comparação a outros instrumentos, principalmente no que se refere à possibilidade de comparabilidade entre os estudos.

As informações obtidas de idosos sobre peso e altura também são menos válidas para estimar o IMC, pois idosos costumam aferir estas medidas com menor freqüência do que os adultos, havendo maior chance de subestimar o IMC ${ }^{42}$. Neste estudo, utilizamos as categorias de IMC recomendadas para idosos, com pontos de corte que levam em consideração as mudanças na composição corporal decorrentes do envelhecimento 23 . 
Por fim, uma vez que as amostras foram planejadas considerando a estimação de proporções, os testes estatísticos foram realizados sem controle do poder do teste, sendo este fato também uma limitação do presente estudo.

Levando-se em consideração que o lazer, pela definição de Dumazedier 56 (p. 29), refere-se a um conjunto de ações escolhidas pelo sujeito para "repousar.. divertir-se, recrear-se e entreterse ou, ainda para desenvolver sua informação ou formação desinteressada, sua participação social voluntária ou sua livre capacidade criadora, após livrar-se ou desembaraçar-se das obrigações pro- fissionais, familiares e sociais", é necessário que as políticas públicas direcionem e incentivem ações mais "ativas", como a prática de exercícios e de esporte contrapondo ao vazio do repouso e da recuperação física e às atividades passivas como o assistir à televisão no espaço de tempo do lazer, principalmente nos segmentos menos favorecidos economicamente e nos idosos com mais idades. É importante também, integrar ao incentivo às práticas corporais outras ações de promoção da saúde, para assegurar maior adesão e eqüidade na incorporação de comportamentos saudáveis.

\section{Resumo}

O propósito deste estudo foi medir a prevalência e os fatores associados à prática de atividade física global e de lazer em idosos (60 anos e mais). Estudo transversal de base populacional, com amostragem em múltiplos estágios que envolveu 1.950 idosos, residentes em áreas do Estado de São Paulo. A prevalência de atividade física global (avaliada pela versão curta do Questionário Internacional de Atividade Física - QIAF) foi de $73,9 \%$ e no lazer de $28,4 \%$. Os resultados apontam para diferenças entre os fatores associados à atividade física global e no lazer. Os segmentos sociais mais vulneráveis ao sedentarismo global e, em especial ao sedentarismo no contexto de lazer, devem ser os alvos preferenciais das políticas de saúde que buscam a promoção de estilos de vida mais saudáveis.

Atividade Motora; Atividades de Lazer; Saúde do Idoso

\section{Colaboradores}

M. P. A. Zaitune realizou a proposta do artigo, a revisão da literatura, a análise dos dados e a redação do artigo. M. B. A. Barros orientou a proposta do artigo, a análise dos dados e a redação do texto, bem como participou do desenvolvimento do projeto ISA-SP, da elaboração dos instrumentos, coordenou a pesquisa de campo. C. L. G. César, L. Carandina, M. Goldbaum e M. C. G. P. Alves contribuíram no desenvolvimento do projeto ISA-SP, na elaboração dos instrumentos, coordenaram a pesquisa de campo e contribuíram na revisão do artigo.

\section{Agradecimentos}

À Fundação de Amparo à Pesquisa do Estado de São Paulo (FAPESP) - Projeto de Políticas Públicas, processo no. 88/14099 e à Secretaria Estadual de Saúde de São Paulo pelo financiamento do trabalho de campo. À Secretaria de Vigilância em Saúde do Ministério da Saúde pelo suporte financeiro para a análise dos dados por meio do Centro Colaborador em Análise de Situação de Saúde da Faculdade de Ciências Médicas, Universidade Estadual de Campinas. 


\section{Referências}

1. Hardy S, Grogan S. Preventing disability through exercise: investigating older adults' influences and motivations to engage in physical activity. J Health Psychol 2009; 14:1036-46.

2. Veras R. Envelhecimento populacional contemporâneo: demandas, desafios e inovações. Rev Saúde Pública 2009; 43:548-54.

3. Alves LC, Leite IC, Machado CJ. Perfis de saúde dos idosos no Brasil: análise da Pesquisa Nacional por Amostra de Domicílios de 2003 utilizando o método grade of membership. Cad Saúde Pública 2008; 24:535-46.

4. Malta DC, Castro AM, Gosch CS, Cruz DKA, Bressan A, Nogueira JD, et al. A Política Nacional de Promoção da Saúde e a agenda da atividade física no contexto do SUS. Epidemiol Serv Saúde 2009; 18:65-78.

5. Vogel T, Brechat PH, Lepretre PM, Kaltenbach G, Berthel M, Lonsdorfer J. Health benefits of physical activity in older patients: a review. Int J Clin Pract 2009; 63:303-20.

6. Adamoli AN, Azevedo MR. Padrões de atividade física de pessoas com transtornos mentais e de comportamento. Ciênc Saúde Coletiva 2009; 14:243-51.

7. Nelson ME, Rejeski WJ, Blair SN, Duncan PW, Judge JO, King AC, et al. Physical activity and public health in older adults: recommendation from the American College of Sports Medicine and the American Heart Association. Med Sci Sports Exerc 2007; 39:1435-45.

8. Phoenix C, Grant B. Expanding the agenda for research on the physically active aging body. J Aging Phys Act 2009; 17:362-79.

9. Aoyagi Y, Park H, Watanabe E, Park S, Shephard RJ. Habitual physical activity and physical fitness in older Japanese adults: the Nakanojo Study. Gerontology 2009; 55:523-31.

10. Assumpção CO, Pellegrinotti I, Bartholomeu Neto J, Montebelo M. Controle da intensidade progressiva de exercícios localizados em mulheres idosas por meio da percepção subjetiva de esforço (BORG). Rev Educ Fís 2008; 19:33-9.

11. Guirao-Goris JA, Cabrero-García J, Moreno-Pina JP, Muñoz-Mendoza CL. Revisión estructurada de los cuestionarios y escalas que miden la actividad física en los adultos mayores y ancianos. Gac Sanit 2009; 23:51-67.

12. Sallinen J, Leinonen R, Hirvensalo M, Lyyra TM, Heikkinen E, Rantanen T. Perceived constraints on physical exercise among obese and non-obese older people. Prev Med 2009; 49:506-10.

13. Cassou ACN, Fermino RC, Santos MS, RodriguezAñez CR, Reis RS. Barreiras para a atividade física em idosos: uma análise por grupos focais. Rev Educ Fís 2008; 19:353-60.

14. Taddei CF, Ramos LR, Moraes JC, Wajngarten M, Libberman A, Santos SC, et al. Estudo multicêntrico de idosos atendidos em ambulatórios de cardiologia e geriatria de instituições brasileiras. Arq Bras Cardiol 1997; 69:327-33.
15. Baretta E, Baretta M, Peres KG. Nível de atividade física e fatores associados em adultos no Município de Joaçaba, Santa Catarina, Brasil. Cad Saúde Pública 2007; 23:1595-602.

16. Siqueira FV, Facchini LA, Piccini RX, Tomasi E, Thumé E, Silveira DS, et al. Atividade física em adultos e idosos residentes em áreas de abrangência de unidades básicas de saúde de municípios das regiões Sul e Nordeste do Brasil. Cad Saúde Pública 2008; 24:39-54.

17. César CLG, Carandina L, Alves MCGP, Barros MBA, Goldbaum M, organizadores. Saúde e condição de vida em São Paulo: Inquérito Multicêntrico de Saúde no Estado de São Paulo (ISA-SP). v. 1. São Paulo: Faculdade de Saúde Pública, Universidade de São Paulo; 2005.

18. Alves MCGP. Plano de amostragem. In: César CLG, Carandina L, Alves MCGP, Barros MBA, Goldbaum M, organizadores. Saúde e condição de vida em São Paulo: Inquérito Multicêntrico de Saúde no Estado de São Paulo (ISA-SP). v. 1. São Paulo: Faculdade de Saúde Pública, Universidade de São Paulo; 2005. p. 47-62.

19. Benedetti TRB, Antunes PC, Añez CRR, Mazo GZ, Petroski EL. Reprodutibilidade e validade do Questionário Internacional de Atividade Física (IPAQ) em homens idosos. Rev Bras Med Esporte 2007; 13:11-6.

20. Abu-Omar K, Rütten A. Relation of leisure time, occupational, domestic, and commuting physical activity to health indicators in Europe. Prev Med 2008; 47:319-23.

21. Matsudo S, Araújo T, Matsudo V, Andrade D, Andrade E, Oliveira LC, et al. Questionário Internacional de Atividade Física (IPAQ): estudo de validade e reprodutibilidade no Brasil. Rev Bras Ativ Fís Saúde 2001; 6:5-18.

22. World Health Organization. A user's guide to the Self Reporting Questionnaire. Geneva: World Health Organization; 1994.

23. Cervi A, Franceschni SCC, Priore SE. Análise crítica do uso do índice de massa corporal para idosos. Rev Nutr 2005; 18:765-75.

24. Francisco PMSB, Donalísio MR, Barros MBA, César CLG, Carandina L, Goldbaum M. Medidas de associação em estudo transversal com delineamento complexo: razão de chances e razão de prevalência. Rev Bras Epidemiol 2008; 11:347-55.

25. Barbosa AR, Lebrão ML, Marucci MFN. Prevalência de inatividade física em idosos do Município de São Paulo. Lecturas: EF y Deportes 2007; 11(105). http://www.efdeportes.com/efd105/prevalenciade-inatividade-fisica-em-idosos.htm.

26. Benedetti TRB, Borges LJ, Petroski EL, Gonçalves LHT. Atividade física e estado de saúde mental de idosos. Rev Saúde Pública 2008; 42:302-7.

27. Pitanga FJG, Lessa I. Prevalência e fatores associados ao sedentarismo no lazer em adultos. Cad Saúde Pública 2005; 21:870-7.

28. Varo JJ, Martínez-González MA, Irala-Estévez J, Kearney J, Gibney M, Martínez JA. Distribuion and determinants of sedentary lifestyles in the European Union. Int J Epidemiol 2003; 32:138-46. 
29. Malta DC, Moura EC, Castro AM, Cruz DKA, Morais Neto OL, Monteiro CA. Padrão de atividade física em adultos brasileiros: resultados de um inquérito por entrevistas telefônicas, 2006. Epidemiol Serv Saúde 2009; 18:7-16.

30. Azevedo MR, Araújo CL, Reichert FF, Siqueira FV, Silva MC, Hallal PC. Gender differences in leisuretime physical activity. Int J Public Health 2007; 52:8-15.

31. Salles-Costa R, Heilborn ML, Werneck GL, Faerstein E, Lopes CS. Gênero e prática de atividade física de lazer. Cad Saúde Pública 2003; 19 Suppl 2:S325-33.

32. Hallal PC, Matsudo SM, Matsudo VKR, Araújo TL, Andrade DR, Bertoldi AD. Physical activity in adults from two Brazilian areas: similarities and differences. Cad Saúde Pública 2005; 21:573-80.

33. Pitsavos C, Panagiotakos DB, Lentzas Y, Stefanadis C. Epidemiology of leisure-time physical activity in socio-demographic, lifestyle and psychological characteristics of men and women in Greece: the ATTICA study. BMC Public Health 2005; 5:37.

34. Santos R, Silva P, Santos P, Ribeiro JC, Mota J. Physical activity and perceived environmental attributes in a sample of Portuguese adults: results from the Azorean Physical Activity and Health study. Prev Med 2008; 47:83-8.

35. Seclén-Palacín JA, Jacoby ER. Factores sociodemográficos y ambientales asociados com la actividad física deportiva en la población urbana del Peru. Rev Panam Salud Pública 2003; 14:255-64.

36. Oehlschlaeger MHK, Pinheiro RT, Horta B, Gelatti C, San'tana P. Prevalência e fatores associados ao sedentarismo em adolescentes de área urbana. Rev Saúde Pública 2004; 38:157-63.

37. Guimarães VMV, César CGL. Atividade física. In: César CLG, Carandina L, Alves MCGP, Barros MBA, Goldbaum M, organizadores. Saúde e condição de vida em São Paulo: Inquérito Multicêntrico de Saúde no Estado de São Paulo (ISA-SP). v. 1. São Paulo: Faculdade de Saúde Pública, Universidade de São Paulo; 2005. p. 91-101.

38. Camões M, Lopes C. Fatores associados à atividade física na população portuguesa. Rev Saúde Pública 2008; 42:208-16

39. Yancey AK, Wold CM, McCarthy WJ, Weber MD, Lee B, Simon PA, et al. Physical inactivity and overweight among Los Angeles county adults. Am J Prev Med 2004; 27:146-52.

40. Hallal PC, Victora CG, Wells JC, Lima RC. Physical inactivity: prevalence and associated variables in Brazilian adults. Med Sci Sports Exerc 2003; 35:1894-900

41. Standridge JB, Zylstra RG, Adams SM. Alcohol consumption: an overview of benefits and risks. South Med J 2004; 97:664-72.

42. Silveira EA, Araújo CL, Gigante DP, Barros AJD, Lima MS. Validação do peso e altura referidos para o diagnóstico do estado nutricional em uma população de adultos no Sul do Brasil. Cad Saúde Pública 2005; $21: 235-45$.
43. Blair SN. Physical activity, epidemiology, public health and the American College of Sports Medicine. Med Sci Sports Exerc 2003; 35:1463.

44. Bowles HR, FitzGerald SJ, Morrow Jr. JR, Jackson AW, Blair SN. Construct validity of self-reported historical physical activity. Am J Epidemiol 2004; 160:279-86.

45. Monteiro CA, Conde WL, Matsudo SM, Matsudo VR, Bonseñor IM, Lotufo PA. A descriptive epidemiology of leisure-time physical activity in Brazil, 1996-1997. Rev Panam Salud Pública 2003; 14: 246-54.

46. Mukamal KJ, Ding EL, Djoussé L. Alcohol consumption, physical activity, and chronic disease risk factors: a population-based cross-sectional survey. BMC Public Health 2006; 6:118.

47. Woo J, Ho SC, Yu AL. Lifestyle factors and health outcomes in elderly Hong Kong Chinese aged 70 years and over. Gerontology 2002; 48:234-40.

48. Willett W. Anthropometric measures and body composition. In: Willett W, editor. Nutritional epidemiology. 2nd Ed. New York: Oxford University Press; 1998. p. 245-7.

49. Guimarães JMN, Caldas CP. A influência da atividade física nos quadros depressivos de pessoas idosas: uma revisão sistemática. Rev Bras Epidemiol 2006; 9:481-92.

50. Moraes H, Deslandes A, Ferreira C, Pompeu FAMS, Ribeiro P, Laks J. O exercício físico no tratamento da depressão em idosos: revisão sistemática. Rev Psiquiatr Rio Gd Sul 2007; 29:70-9.

51. Hallal PC, Dumith SC, Bastos JP, Reichert FF, Siqueira FV, Azevedo MR. Evolução da pesquisa epidemiológica em atividade física no Brasil: uma revisão sistemática. Rev Saúde Pública 2007; 41: 453-60.

52. Rzewnicki R, Vanden Auweele Y, De Bourdeaudhuij I. Addressing overreporting on the International Physical Activity Questionnaire (IPAQ) telephone survey with a population sample. Public Health Nutr 2003; 6:299-305.

53. Graff-Iversen S, Anderssen SA, Holme IM, Jenum AK. An adapted version of the long International Physical Activity Questionnaire (IPAQ-L): construct validity in a low-income, multiethnic population study from Oslo, Norway. Int J Behav Nutr Phys Act 2007; 4:13.

54. Motl RW, McAuley E, DiStefano C. Is social desirability associated with self-reported physical activity? Prev Med 2005; 40:735-9.

55. Benedetti TRB, Mazo GZ, Barros MV. Aplicação do Questionário Internacional de Atividade Física para avaliação do nível de atividades físicas de mulheres idosas: validade concorrente e reprodutibilidade teste/reteste. Rev Bras Ciênc Mov 2004; 12:25-33.

56. Dumazedier J. Sociologia empírica do lazer. São Paulo: Editora Perspectiva; 2001.

Recebido em 19/Abr/2009

Versão final reapresentada em 05/Abr/2010 Aprovado em 03/Mai/2010 